Teknokultura. Revista de Cultura Digital y Movimientos Sociales

ISSNe: $1549-2230$

http://dx.doi.org/10.5209/TEKN.63104

\title{
Política en las redes, redes en la política: estudio de caso de la campaña \#VivirSinMiedo
}

\author{
Santiago Escuder ${ }^{1}$
}

Recibido: 31 de enero de 2019 / Aceptado: 21 de marzo de 2019 Open peer reviews

Resumen. Esta investigación se propuso explorar la campaña de reforma constitucional "Vivir Sin Miedo" como estudio de caso sobre el uso de las redes sociales electrónicas a nivel político en Uruguay. La reforma considera entre otros puntos la creación de una guardia militar y la "cadena perpetua revisable". El objetivo del trabajo es mostrar cómo los diferentes actores nucleados en torno a la temática captan y se apropian del espacio virtual. Conocer la forma topológica de estas redes y vislumbrar si estas logran despolitizar el tema e impactar en la difusión de su mensaje hacia nuevos adherentes de todos los partidos políticos. O por el contrario, representan una red compacta de actores dentro de un mismo espectro ideológico, que comparten una misma preocupación sin ir más allá de sus preferencias partidarias. Para ello, se colectaron un conjunto de tweets y retweets en torno al hashtag \#VivirSinMiedo" para su posterior procesamiento y análisis en grafos. Entre los principales hallazgos se destacan la existencia de una red compacta descentralizada en tres grandes sub-redes, las cuales están conformadas básicamente por los propulsores de la campaña por un lado (núcleo duro de actores políticos vinculados a la reforma). Un cinturón de adherentes partidarios. Y otro cinturón de adherentes no partidarios, con lazos débiles hacia los actores políticos. Pese a existir "brokers" y replicadores de información independientes (periodistas), la red \#VivirSinMiedo no hace más que difundir la campaña entre "iguales" (nodos que comparten preferencias políticas), siendo su capacidad de expansión hacia nuevos adherentes muy restringida.

Palabras clave: activismo digital; análisis de Redes; inseguridad ciudadana; Twitter.

\section{[en] Policy in networks, networks in policy. Case study of the campaign \#VivirSinMiedo}

Abstract. This research project aims to explore the campaign for constitutional reform known as
"Live Without Fear" as a case study on the use of electronic social networks at a political level in
Uruguay. Among other points, the proposed amendment considers the creation of a military guard and
the institution of "revisable life imprisonment". The objective of this piece is to show how the different
actors gathered around the theme capture and take ownership of the virtual space. Also to learn about
the topological form of these networks and to see if these are able to depoliticize the issue and impact
on the dissemination of their message to new adherents from all political parties; or if, on the contrary,
they represent a compact network of actors within the same ideological spectrum, who share the same
concern without going beyond their party preferences. To do this, a set of tweets and retweets were
collected around the hashtag \#VivirSinMiedo [\#LiveWithoutFear] for further processing and analysis
in graphs. The main findings include the existence of a compact network decentralized into three large
sub-networks, which are basically made up of the proponents of the campaign on the one hand (hard core
of political actors linked to the amendment campaign); a circle of party adherents, on the other hand;
and another outer-circle of non-party adherents with weak ties to political actors. Despite the existence

$1 \quad$ Universidad de la República (Uruguay)

E-mail: santiago.escuder@cienciassociales.edu.uy 
of independent "brokers" and replicators of information (journalists), the \#VivirSinMiedo network does not do more than spread the campaign among "peers" (nodes that share political preferences), and its ability to expand towards new adherents is very restricted.

Keywords: citizen insecurity; digital activism; network analysis; Twitter.

Sumario. 1. Introducción. 2. Electores y participación política en Internet. 3. La forma de las redes sociales. 4. Redes sociales en Uruguay. Por qué estudiar Twitter como red social electronica. 5. La campaña \#VivirSinMiedo: el problema de la inseguridad y el salvavidas político de Larrañaga. 6. Problema de investigación, preguntas, objetivos e hipótesis. La campaña \#VivirSinMiedo como estudio de caso. 7. Diseño metodológico y técnica. 8. Principales hallazgos. 9. Conclusiones. 10. Referencias.

Cómo citar: Escuder, S. (2019). Política en las redes, redes en la política: estudio de caso de la campaña \#VivirSinMiedo, en Teknokultura. Revista de Cultura Digital y Movimientos Sociales, 16(1), 55-74.

\section{Introducción}

Las redes sociales electrónicas se han configurado como espacios de propaganda y proselitismo político en Uruguay y el mundo. Producto de ello, la captación del espacio virtual construye redes de conexión emergentes entre adherentes partidarios y no tan afines. En este sentido, la difusión de la campaña "Vivir Sin Miedo" no resulta la excepción.

El objetivo principal del trabajo es explorar la red como consecuencia del hashtag \#VivirSinMiedo en la red social electrónica Twitter. Los objetivos específicos se proponen: 1. Conocer el rol especifico del senador Jorge Larrañaga en dicha red; 2. Conocer el perfil de quienes replican el hashtag \#VivirSinMiedo y como se posicionan en la red, especialmente de nuevos actores por fuera del sector de Larrañaga, Alianza Nacional y; 3. Detectar nuevos adherentes de otros partidos o ideologías no afines a la campaña.

\section{Electores y participación política en Internet}

En la estructura mediática de las sociedades "modernas" o "industriales", los actores políticos y muchas de las estructuras institucionales y representativas (sindicatos, gremios, $\mathrm{ONG}^{\prime}$ s, etc.), necesariamente tenían que operar el lenguaje y los códigos reducidos de los medios tradicionales (sobre todo de la televisión), para poder expresar sus ideas e iniciativas al público elector. Lo que comúnmente se denomina mecanismos de democracia participativa, como consultas directas, plebiscitos y referéndums (Reveles Vázquez, 2017), la creación y difusión de la opinión pública sobre determinados temas se veían relegados meramente a canales comunicacionales "unídireccionales" mediados por los medios masivos. Se trataba de una lógica de reproducción comunicacional estilo "top-down", donde el contacto resultaba netamente jerarquizado, tanto entre los mismos actores políticos, como de estos con los ciudadanos, con pocas instancias de intercambio, fundamentalmente en co-presencia.

En la Sociedad de la Información y el Conocimiento (SIC), esta condición gana exponencial pluralismo. Se refuerza la exposición de los representantes, lo que piensan sobre diversas problemáticas del vivir cotidiano. Se dispone de mayor 
información y mayor oferta electoral para poder elegir. Pero lo más importante, aumenta el "feedback" constante entre usuarios y dirigentes, que mediante las nuevas herramientas de la Web 2.0 (blogs, wikis, redes sociales electrónicas, etc.), logran canalizar necesidades y "crear" opinión pública ("agenda setting"). Es decir, la versatilidad, el sencillo manejo y la velocidad de las TIC permiten transformar la estructura comunicativa hacia un modelo "bottom up" entre líderes políticos y ciudadanos. El beneficio de los partidos políticos y las estructuras representativas no recaen sólo en la amplificación y la resonancia de sus mensajes e ideas, sino también en el monitoreo mediante "microtargeting". Es decir, recaudar información y distinguir perfiles o segmentos de electores, lo cual permite direccionar el discurso de acuerdo a dichas preferencias, distinguir líderes digitales ("influenciadores") o expandir lo que se conoce como las narrativas políticas transmedia (Orlando D’Adamo, et. al. 2015; Gerl, 2017).

Como consecuencia de ello, los monopolios de la información se pierden. Tanto ciudadanos como políticos tienen las mismas oportunidades de enfrentarse en el espacio virtual y recabar información mediante foros de debate, plataformas específicas, redes sociales electrónicas, sin la intermediación que imponía los medios tradicionales o los contingentes territoriales (Ferras, et al. 2009; Toudert, 2014; Marín Dueñas y Díaz Guerra, 2016).

Sin embargo, este nuevo pluralismo virtual no genera necesariamente electores más informados, o políticos más críticos de sus ideas. Por el contrario, la instantaneidad de los discursos puede conducir a la desinformación generalizada ("noticias falsas"), expansión de discursos contradictorios, sub-información, o propaganda y manipulación que inhibe a la razón, además de compromisos poco duraderos (Otaola, 2007). Bien como mencionan Batlle y Cerrillo (2004), el "ignorante racional" no se esfuerza en buscar información, sino visualiza algún tipo de beneficio. El mismo desinterés que los electores experimentaban off-line, también se reproduce en Internet. Pero también del lado de los actores políticos, el sostener y dedicarle energía a estos medios puede conducir a la verborragia o catarsis improductiva. Por ejemplo, mensajes irreflexivos, sin ninguna propuesta u objetivo inherente más que marcar presencia o estar en la red. Ello puede producir la desconfianza de los ciudadanos, quienes cuestionen el tiempo efectivamente que dedican los funcionarios políticos y representantes institucionales a sus verdaderas funciones (Lusoli y Ward, 2003).

\section{La forma de las redes sociales}

Varias son las plataformas y aplicaciones de comunicación y difusión de información que pululan en la red. Pero Internet también se ha configurado como un "medio de medios" absorbiendo formas tradicionales de comunicación, como la radio, la televisión, la industria cinematográfica y audiovisual, entre otras. A este fenómeno se lo ha denominado "ubicuidad" digital (Islas, 2008; Castro Rojas, 2012), y no es más que la fusión y presencia de varios medios en uno sólo, en dispositivos fundamentalmente móviles, al instante y en cualquier lugar.

Entre las aplicaciones más populares y utilizadas, se encuentran las redes sociales electrónicas, que paulatinamente han ido sustituyendo a muchas formas tradicionales de comunicación dentro de Internet. Por ejemplo, los foros de intercambio de materiales, el correo electrónico, los medios audiovisuales, entre otros, confluyen 
todos juntos en estas nuevas redes virtuales, como Facebook, Youtube, Instagram o Twitter. Sin embargo, la existencia de las redes sociales como tales, data de mucho antes del nacimiento de Internet y de la conformación del espacio virtual tal como lo conocemos. De allí su importancia en comprender la naturaleza de su estructura, además de los flujos de comunicación e información que portan sus distintos integrantes (nodos).

Existen múltiples formas topológicas de redes sociales. No obstante, Paul Baran (1964), uno de los primeros precursores del estudio de redes, reconoce tres grandes tipos prevalecientes (ver figura $\mathrm{n}^{\circ} 1$ ). Las "redes centralizadas", donde todos los vínculos recaen en un nodo central, siendo el resto de nodos periféricos. Esta red también es conocida como red estrella o red egocéntrica (Hogan, 2017). Las "redes descentralizadas", las cuales a diferencia de la red anterior, se clasifican en varios grupos que conectan a varios nodos centrales, que a su vez conectan a un nodo central y; la "red distribuida", la cual no reconoce nodos centrales, sino que todos se interconectan de tal modo que ninguno es imprescindible en la red.

Imagen 1. Modelos de redes centralizada, descentralizada y distribuida. Fuente: P. Baran (1964)²
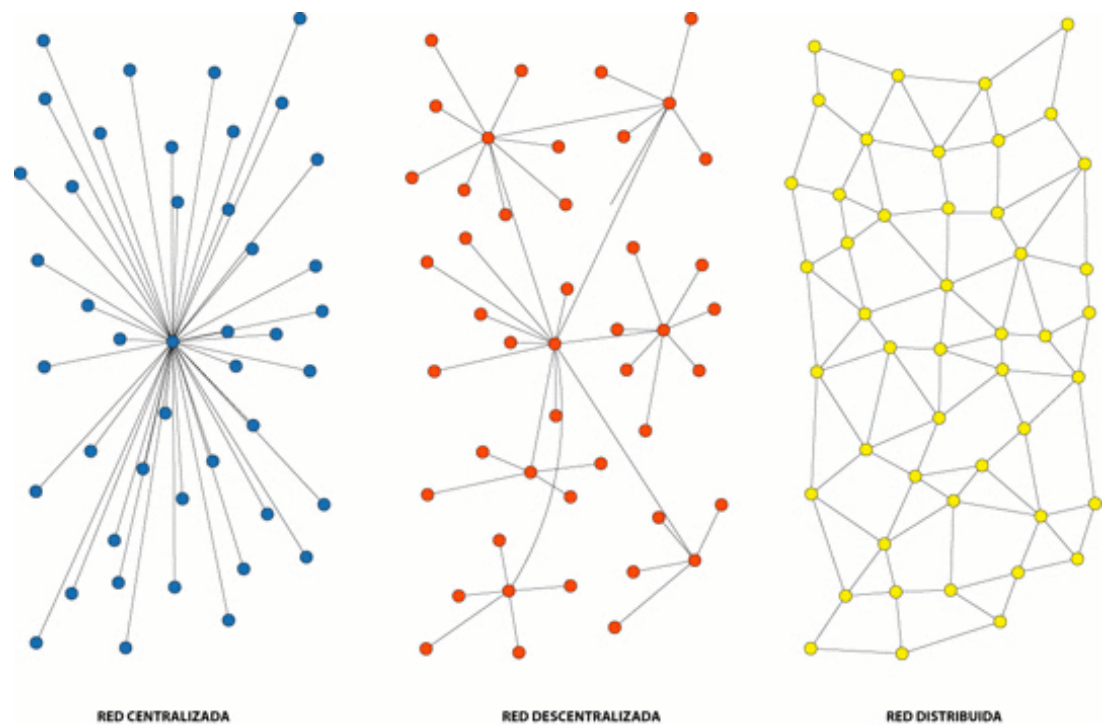

También es importante remarcar ciertos elementos que hacen a la cohesión y conexión (densidad) de las redes, así como la transitividad entre los nodos (Freeman, 1979). Es decir, si existen lazos de un supuesto actor A hacia el B y del B a otro actor $\mathrm{C}$, debería de existir una conexión entre $\mathrm{C}$ y A. En pocas palabras, "los amigos de mis amigos son mis amigos". Este principio de gustos compartidos, pero también de antipatía, Moreno (1934) lo denominó como "tele”, y no es más que la causa de elección, transferencia hacia el otro, o rechazó mutuo interpersonal. 2 https://www.rand.org/content/dam/rand/pubs/research_memoranda/2006/RM3420.pdf (fecha de consulta, 
La conexión y cercanía de los integrantes de una red corresponden a lazos fuertes que pueden conformar comunidades de nodos (clusters). Sin embargo, pese a que los nodos interconectados disminuyen la distancia promedio global de la red en su conjunto, no pueden proporcionar conexiones más allá del universo que manejan los conglomerados. En contrapartida, los lazos débiles juegan un papel preponderante como conectores hacia la "diversidad" (afuera de los lazos y clusters con mayor transitividad), proporcionando muchas veces información novedosa y nuevos contactos. Muchas veces estos nodos figuran como puente, llamado "bróker", los cuales figuran como intermediarios entre dos o varios grupos, teniendo muchos lazos débiles entre redes, pero con un rol central en conectarlas. En este sentido, la red puede contener huecos o agujeros estructurales, es decir, desconexión entre grupos de nodos (Burt, 2004). Si bien esto puede ser un problema, los roles de intermediación (nodos brokers) pueden tener un papel preponderante en esta falencia.

\section{Redes sociales en Uruguay. Por qué estudiar Twitter como red social electrónica}

Dentro de la región latinoamericana, Uruguay se encuentra en la vanguardia en acceso y uso de las nuevas Tecnologías de la Información y la Comunicación (TIC). De acuerdo al estudio "El perfil del Internauta Uruguayo" realizando en el año 2018 por la consultora Grupo Radar ${ }^{3}$, el 73\% de los hogares contaba con conexión a Internet, siendo el $89 \%$ de los uruguayos usuarios de Internet. El celular fue el dispositivo más utilizado por las personas para conectarse a Internet (84\%).

De acuerdo a estadísticas oficiales, según la Encuesta de Usos de Tecnologías de la Información y la Comunicación (EUTIC) realizada por el Instituto Nacional de Estadística para el año 2016, el 74\% de las personas también era usuaria regular de Internet ${ }^{4}$.

Entre los principales usos de Internet realizados por las personas según Radar figuran "Buscar información de Interés, "Utilizar redes sociales electrónicas, "Chatear por redes sociales. Casi la mitad de los usuarios utilizaban Internet para entrar a redes sociales electrónicas como su principal fin. Según la EUTIC, Facebook es la principal red con un $60 \%$ de los usuarios, seguidos por Instagram (21\%) y Twitter $(17 \%)^{5}$.

En cuanto al uso de redes sociales electrónicas en campañas políticas, Luis Alberto Lacalle Herrera fue uno de los primeros políticos en utilizar Facebook de manera activa en la campaña por las elecciones del año 2009. Utilizó su cuenta básicamente para informar sobre eventos del partido Nacional (centro derecha), invitando a los uruguayos (especialmente a jóvenes) a formular propuestas y plantear preguntas. Algo similar ocurrió con Pedro Bordaberry (partido Colorado, centro derecha) en Twitter. Si bien el oficialismo (Frente Amplio) contaba con militantes digitales ("Redes Frenteamplistas"), los principales candidatos no utilizaban las redes sociales electrónicas, pese a existir cuentas no oficiales, que apoyaban las candidaturas de José Mujica y Danilo Astori (Radakovich, et. al, 2013).

\footnotetext{
Por más información ver: http://www.gruporadar.com.uy/01/?p=2377 (fecha de consulta, 25/08/2018)

Ver informe en https://www.agesic.gub.uy/innovaportal/file/6308/1/eutic2016_final.pdf (fecha de consulta, 25/08/2018)

5 http://www.ine.gub.uy/c/document_library/get_file?uuid=21edeb0d-0ae1-4bc8-b9a37d515f077228\&groupId=10181 (fecha de consulta, 25/08/2018)
} 
A pesar de que Twitter es la red con menor penetración entre los usuarios de Internet, destaca entre los principales candidatos de la oposición al gobierno, los cuales cuentan con la mayor cantidad de seguidores, para las dimensiones de Uruguay, que cuenta con poco más de 3,2 millones de habitantes ${ }^{6}$. Luis Lacalle Pou con 117 mil seguidores, seguido de Jorge Larrañaga (105 mil seguidores) y Verónica Alonso (43,3 mil) del partido Nacional. Dentro del Partido Colorado, los candidatos José Amorín Batlle (31,4 mil seguidores), Ernesto Talvi (27,8 mil seguidores), y el ex presidente Julio María Sanguinetti (13,3 mil) son los que acarrean más seguidores. Dentro del Partido Independiente, Pablo Mieres lo siguen unos 32,6 seguidores ${ }^{7}$.

El caudal de seguidores es mucho menor dentro de los candidatos del gobierno. Daniel Martínez cuenta con 70,7 mil seguidores, seguido por Carolina Cosse $(14,4$ mil) y Mario Vergara (1.780) Oscar Andrade no tiene una cuenta personal en Twitter, sino una de campaña con 777 seguidores $^{8}$.

¿Por qué estudiar el desempeño en Twitter y no en otra red social electrónica? Las redes sociales permiten la interrelación entre los usuarios, siendo ellos mismos "prosumidores" de sus propios contenidos. Resulta una herramienta portable, de sencillo manejo, gratuita y democrática si se dispone de conexión a Internet. Además, al igual que el resto de las redes, integran todos los contenidos multimedia existentes. Permite esquivar los medios tradicionales para comunicarse con el electorado de manera instantánea (Marín Dueñas y Díaz Guerra, 2016).

Por otra parte, y contrario a Facebook, la dinámica propia de Twitter de "microbloging" permite "fortalecer la escritura", generando capacidad de síntesis de los mensajes que se quieren difundir en sólo 140 caracteres, siendo una virtud para los influenciadores y generadores de opinión pública, entre ellos los actores políticos (Fainholc, 2011). Además, no sólo mantiene el contacto con los electores, sino también con los detractores, fomentando el debate de ideas y la discusión política (Salgado, 2013).

¿Por qué no estudiar Twitter? Entre sus principales críticas, Fainholc (2011) reconoce como principales problemas la adicción a la herramienta (el estar constantemente "pendiente" o "conectado"), genera un efecto disruptivo en la escritura, que configura un pensamiento simple y acrítico reducido en 140 caracteres, que en muchos casos fomenta la política banal y las noticias efímeras. Ello implica cuestionar el intercambio veraz de información que se produce en la red, el cual muchas veces se aleja de una instancia educativa y de aprendizaje, para formar parte del tiempo de entretenimiento y ocio virtual de las personas. Por último, y como otras redes sociales, depende de los intereses de grandes corporaciones económicas.

\section{La campaña \#VivirSinMiedo: el problema de la inseguridad y el salvavidas político de Larrañaga}

Para reformar aspectos de la Constitución Nacional de la República Uruguay y/o aprobar o derogar leyes votadas en el parlamento, la misma Constitución contempla un mecanismo específico mediante referéndum (votación de la mitad más uno de

\footnotetext{
Datos disponibles en: http://www5.ine.gub.uy/censos2011/index.html (fecha de consulta, 09/02/2019)

Consulta de cuentas $(10 / 02 / 2019)$

Consulta de cuentas $(10 / 02 / 2019)$
} 
los electores), el cual ha sido utilizado por todos los partidos políticos de Uruguay en diferentes períodos. Se trata en primera instancia de juntar un $25 \%$ de firmas del cuerpo electoral, para luego convocar a consulta por plebiscito de todo el cuerpo electoral (ley $\left.\mathrm{n}^{\circ} 17.244\right)^{9}$.

Varias han sido las instancias en las que se plebiscitaron leyes desde el retorno de la democracia. Para citar algunos ejemplos, por referéndum en el año 1992 bajo el gobierno de Luis Alberto Lacalle Herrera se bloqueó la venta de partes de las acciones de las empresas públicas como la Administración Nacional de Telecomunicaciones, ANTEL (ley n ${ }^{\circ}$ 16.211). Y más recientemente en el año 2003 por la Administración Nacional de Combustibles, Alcohol y Portland, ANCAP (ley $\mathrm{n}^{\circ}$ 17.448). También se mantuvo el monopolio del agua potable en Obras Sanitarias del Estado, OSE. Estos referéndums fueron apoyados por el Frente Amplio cuando fue oposición gubernamental, incrementando de manera elocuente su capital político Otros intentos fueron el referéndum sobre la Ley de Caducidad por la pretensión punitiva del Estado, donde actualmente bajo el artículo $\mathrm{n}^{\circ} 15.848$ se ampara a militares exentos de declarar frente a la justicia. Sin embargo, la reforma fracaso con él $47,98 \%$ de los votos.

Recientemente en el año 2015 también se llevó a plebiscito la propuesta por bajar la edad de imputabilidad de 18 a 16 años. Está reforma fue apoyada por una parte del Partido Nacional y prácticamente todo el Partido Colorado. Su principal propulsor fue el senador Pedro Bordaberry ("Vamos Uruguay"), el cual baso parte de su narrativa discursiva en la inseguridad que asola actualmente al país. Su eslogan fue para "Vivir en paz". La reforma por referéndum no fue aprobada, pese a que contó con el $46,81 \%$ de los votos.

Actualmente el senador Jorge Larrañaga (Partido Nacional) y parte de su aparato político ("Alianza Nacional") juntan firmas para llevar a plebiscito la reforma de tres polémicos artículos de la Constitución: cambios en el artículo $\mathrm{n}^{\circ} 11$ de la Constitución vigente ${ }^{10}$ permitiendo los allanamientos nocturnos; modificaciones al artículo $\mathrm{n}^{\circ} 27$ estableciendo el cumplimiento total de las condenas y la cadena perpetua y (el que se considera más polémico); agregar al artículo n ${ }^{\circ} 85$ la creación de una guardia nacional con integrantes de las Fuerzas Armadas. Es decir, esta última modificación pretende integrar al cuerpo policial soldados militares ${ }^{11}$. La campaña inicio formalmente con un acto en el parlamento el 23/05/2018

Los principales detractores de la reforma alegan retroceder a tiempos de predictadura, donde precisamente se crearon las fuerzas conjuntas (policías y militares), las que luego tomaron el control del Estado, que derivaron en la dictadura cívicomilitar (1973-1985).

Al igual que otros intentos de referéndum, no es casual que Larrañaga busque tomar protagonismo en la agenda política e incrementar su capital político. Vale destacar que dentro del Partido Nacional, según varias encuestas de Opinión Publica, las preferencias en la interna partidaria para Larrañaga $(28 \%)$ se ubica muy por debajo

\footnotetext{
9 Para más información se encuentra disponible en la web la ley n 17.244: https://legislativo.parlamento.gub.uy/ temporales/leytemp3767313.htm (fecha de consulta, 25/08/2018)

10 Constitución de 1967, con las modificaciones plebiscitarias de los años 1989, 1994, 1996 y 2004. Disponible en: https://parlamento.gub.uy/documentosyleyes/constitucion (fecha de consulta 10/02/2019)

11 Para más información: http://vivirsinmiedo.com.uy/

12 Para más detalles consultar https://www.elpais.com.uy/informacion/politica/larranaga-inicia-campana-recoleccion-firmas-reforma-seguridad.html (fecha de consulta 10/02/2019
} 
de Luis Lacalle Pou $(53 \%)^{13}$, principal senador de la oposición (Herrerismo, sector "Todos").

La campaña "Vivir sin Miedo" busca poner a Larrañaga nuevamente como posible candidato para arrebatarle el poder al Frente Amplio, o al menos conseguir reformar la Constitución. Pese a ello, Larrañaga no se muestra partidario de "politizar" estas reformas, fundamentando el apoyo a la propuesta sobre la base de que la inseguridad que vive el país no tiene "color político", procurando recolectar firmas entre toda la ciudadanía. Varias encuestas muestran que más de un $60 \%$ de los adherentes del Partido Nacional, así como del Partido Colorado están dispuestos a firmar para plebiscitar estas reformas. Dentro de los votantes del Frente Amplio esta cifra alcanza solo al $10 \%{ }^{14}$.

En cuanto a actores políticos parlamentarios por fuera del sector Alianza Nacional, adhirieron varios diputados y senadores colorados, como Pedro Bordaberry. Actualmente Larrañaga ya alcanzó las firmas necesarias para realizar el referendúm, más de $370.000^{15}$, pese a seguir recolectando adherentes para ejercer presión sobre el gobierno nacional.

El hashtag \#VivirSinMiedo es parte de la campaña en la red social electrónica Twitter, para difundir el apoyo y captar adherencias de ciudadanos.

\section{Problema de investigación, preguntas, objetivos e hipótesis. La campaña \#VivirSinMiedo como estudio de caso}

La captación del espacio virtual en Internet no es ajena cuando se trata de debates políticos para sumar adherentes de diversos segmentos de la ciudadanía. Estudiar la puja y difusión de ideas políticas en las redes sociales electrónicas, resulta más que pertinente para analizar las narrativas políticas de los diferentes actores y sus posicionamientos en dicho debate.

Además, las redes sociales electrónicas permitirían por otro lado aproximarse y entender la nueva arquitectura organizacional de los partidos políticos, en lo que respecta a la difusión, resonancia de su mensaje en Internet, manteniendo lazos y fidelizando a un nuevo público (potenciales electores).

No obstante, como vimos anteriormente, es necesario sostener que las redes sociales se construyen en base a afinidades y adherencias a gustos y sentimientos compartidos entre los sujetos. En pocas palabras, se trata de un conjunto de personas conocidas entre sí, que comparten determinados lazos de preferencia (principio de "tele"). Por lo que pese a que las redes sociales electrónicas tengan una resonancia muchísimo mayor que el espacio en co-presencia, no hacen más que replicar nuestro universo de gustos, reafirmándolo sobre quienes lo comparten, sin necesariamente llegar a un público mayor. Ello resulta paradójico ya que el fin último de estas herramientas digitales en política, es expandir el mensaje entre adherentes y no tan afines.

La tensión entre la amplificación de estas narrativas políticas y la mera reproducción de preferencias es el problema de nuestra investigación. Surge como principal

13 Para más información ver: http://www.gruporadar.com.uy/01/?p=1987 (fecha de consulta, 25/08/2018)

14 Para más información ver encuesta de Opción Consultores: http://www.opcion.com.uy/opinion-publica/?p=2093 (fecha de consulta, 25/01/2019)

15 https://www.subrayado.com.uy/habra-plebiscito-larranaga-obtuvo-mas-370-mil-adhesiones-n521570 (fecha de consulta, 25/01/2019) 
pregunta: ¿La red que se conforma en torno al hashtag \#VivirSinMiedo logra amplificar el mensaje hacia nuevos adherentes, como buscan sus principales propulsores? O por el contrario, ¿representa ideológicamente una red compacta de ciudadanos que comparten está misma preocupación sin ir más allá de sus preferencias partidarias?

Si bien se trata de un trabajo exploratorio, se maneja como hipótesis central que la red de \#VivirSinMiedo no es más que el espejo de las adherencias partidarias de ciudadanos con afinidades políticas similares, pero que se encuentran en el espacio virtual en forma netamente centralizada y egocéntrica. Se parte del supuesto de que pese a los esfuerzos del senador Larrañaga por despolitizar la reforma constitucional y buscar adherentes de otros partidos, el hashtag ("tema") será compartido por aquellos de su misma filiación o espectro político (transitividad política), lo que derivaría en la mayor centralidad de Larrañaga en dicha red.

Cabe señalar que la centralidad ("ego") de Larrañaga en la red resultaría beneficiosa para incrementar su capital político y colocarlo como un actor relevante dentro de la agenda pública. Pero resultaría contraproducente en cuanto al vuelo a-político o desideológico que pretende dar a la campaña para captar nuevos adherentes.

La hipótesis rival sería lo contrario. La despolitización de la red daría a Larrañaga un papel secundario en el conjunto de nodos, viralizándose el hasta \#VivirSinMiedo por igual entre adherentes al Partido Nacional, como en otras comunidades políticas.

\section{Diseño metodológico y técnicas}

El estudio basa su diseño en un análisis transeccional cuantitativo en un momento dado de la campaña de \#VivirSinMiedo en Twitter. Cada uno de los nodos de la red representará una cuenta de usuario Twitter, mientras que el vínculo entre nodos fueron los retweets y menciones del hashtag \#VivirSinMiedo entre usuarios. Para ello, se extrajeron tweets y retweets que mencionaron el hashtag desde 20/07/2018 hasta el 20/08/2018, momento de mayor ebullición de la campaña. La cuenta@vivirsinmiedouy cuenta 2.977 tweets y unos 1.557 seguidores, gran parte de ellos adherentes al partido nacional.

Se utilizó el programa "R-studio" el cual emplea el motor del programa estadístico original "R". Para extraer la información se recurrió a la librería "TwiterR"16. Vale destacar que la extracción por limitación de la API KEY de Twitter sólo permite recabar la información del presente hasta diez días de antigüedad, por lo que es imposible extraer toda la información generada desde el comienzo de la campaña (mes de Mayo,2018). El período de análisis para colectar los tweets se realizó en tres extracciones. Desde el 01/07/2018 al 30/07/2018, momento en el cual la campaña se encontraba en desarrollo intermedio.

Una vez obtenida la información, junto con el programa "Gephi” se procedió a construir una tabla de aristas "Target" y "Source" en la cual vinculó a los usuarios que mencionan o retwettean el hastag \#VivirSinMiedo. Se utilizó el algoritmo de Yifan Hu para visualizar su topología, el cual permitió una mejor comprensión visual de los grupos, además de los coeficientes "grado de entrada" (información y menciones que recibe un nodo de otros), "grado de salida" (información y menciones que realiza un nodo de otros), "cercanía central" (distancia promedio desde un nodo dado

16 Disponible en: https://cran.r-project.org/web/packages/twitteR/twitteR.pdf (fecha de consulta, 25/01/2019) 
a todos los demás nodos en la red) y la "centralidad de intermediación" (frecuencia con la que aparece un nodo en las rutas más cortas entre los nodos de la red).

\section{Principales hallazgos}

Procesados los datos en Gephi, la red de colecciones de tweets y retweets de la campaña \#VivirSinMiedo ascendía a 423 nodos (cuentas de twitter), las cuales tenían 1195 vínculos entre ellas (aristas). En la imagen $n^{\circ} 2$ y la tabla $n^{\circ} 1^{17}$ se puede observar un resumen de la red completa de aquellos usuarios que compartieron el hashtag \#VivirSinMiedo y la matriz de coeficientes de centralidad, cercanía y lejanía de los nodos.

Imagen 2. Red de nodos y aristas de la red de la campaña \#VivirSinMiedo.

Fuente: elaboración propia en base a modelo de nodos y aristas de tweets y retweets de la campaña \#VivirSinMiedo

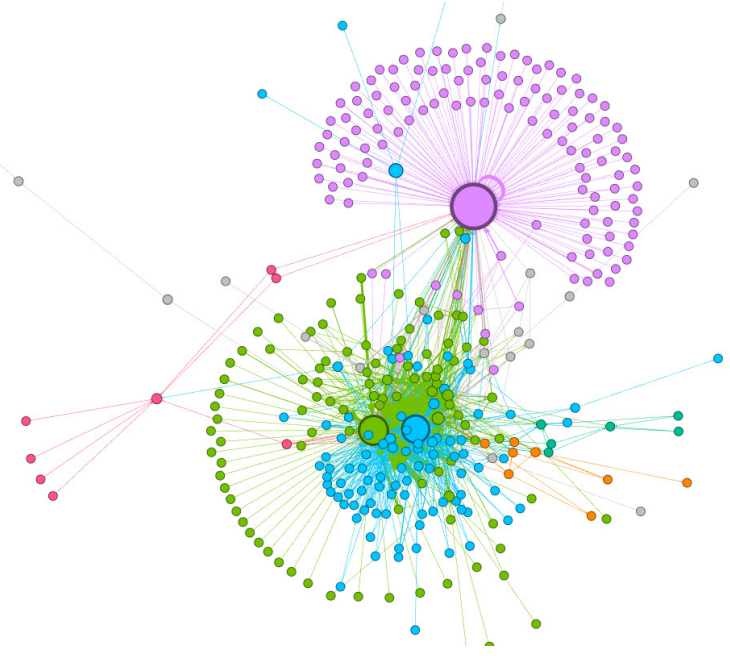

Tabla 1. Coeficientes de grado de entrada, salida

y cercanía de la red de la campaña \#VivirSinMiedo. Fuente: elaboración propia en base a modelo de nodos y aristas de tweets y retweets de la campaña \#VivirSinMiedo

\begin{tabular}{|c|c|c|c|c|}
\hline Cuenta & $\begin{array}{c}\text { Grado de } \\
\text { entrada }\end{array}$ & $\begin{array}{c}\text { Grado de } \\
\text { salida }\end{array}$ & $\begin{array}{c}\text { Cercanía } \\
\text { Central }\end{array}$ & $\begin{array}{c}\text { Centralidad de } \\
\text { Intermediación }\end{array}$ \\
\hline vivirsinmiedouy & 407 & 58 & 0.584639 & 21.818 .764 .966 \\
\hline jorgewlarranaga & 300 & 12 & 0.589258 & 1.992 .496 .987 \\
\hline Usuario_XXX1 & 183 & 14 & 0.588328 & 38.309 .718 .576 \\
\hline
\end{tabular}

17 Mencionamos con un nombre de fantasía ("Usuario_XXX1") cuentas personales de usuarios que no son actores políticos o personalidades públicas a los efectos de mantener el anonimato público 


\begin{tabular}{|c|c|c|c|c|}
\hline Cuenta & $\begin{array}{c}\text { Grado de } \\
\text { entrada }\end{array}$ & $\begin{array}{l}\text { Grado de } \\
\text { salida }\end{array}$ & $\begin{array}{c}\text { Cercanía } \\
\text { Central }\end{array}$ & $\begin{array}{l}\text { Centralidad de } \\
\text { Intermediación }\end{array}$ \\
\hline alianzauy & 132 & 35 & 0.507483 & 3.452 .779 .023 \\
\hline cdcamy & 75 & 0 & 0.433721 & 66.429 .609 \\
\hline PedroBordaberry & 63 & 0 & 0.420993 & 1.814 .099 .088 \\
\hline jorgegandini & 60 & 1 & 0.424829 & 892.183 .813 \\
\hline JuntosPodemos54 & 56 & 9 & 0.420045 & 289.205.021 \\
\hline dipolivera & 48 & 0 & 0.39893 & 288.611 .098 \\
\hline pabloabdala66 & 45 & 13 & 0.482536 & 1.114 .053 .819 \\
\hline DipGarciaMario & 34 & 1 & 0.414444 & 203.563 .252 \\
\hline lista250 & 30 & 4 & 0.455433 & 150.605 .127 \\
\hline JovenesJuntos 36 & 28 & 16 & 0.412155 & 123.889 .436 \\
\hline Lista66 & 22 & 2 & 0.4117 & 21.860 .166 \\
\hline$(\ldots)$ & $(\ldots)$ & $(\ldots)$ & $(\ldots)$ & $(\ldots)$ \\
\hline AlfTuitea & 13 & 0 & 0.282576 & 4398 \\
\hline Usuario_XXX2 & 0 & 5 & 0.383745 & 539.747 .002 \\
\hline
\end{tabular}

Entre los principales resultados, se puede vislumbrar que la topología de la campaña obedece a una red descentralizada en tres grandes grupos ("clústers") que conectan a tres nodos centrales, los cuales reciben el mayor caudal de información. Son muy pocos los nodos que alcanzan a recorrer la red entera de manera genuina ("mencionar" y ser "mencionado"). Por el contrario, nodos muy cercanos y próximos entre sí ideológicamente, muchas veces no presentan ninguna conexión.

La primera red podría definirse como el "núcleo duro" de la campaña de \#VivirSinMiedo (imagen $\mathrm{n}^{\circ} 3 \mathrm{y} \mathrm{n}^{\circ} 4$ ), donde se encuentra la mayor densidad entre cuentas de usuarios. En el centro de esta red se encuentran dos de sus nodos principales. Por un lado, la cuenta del propio movimiento "Vivir Sin Miedo" (@VivirSinMiedo), tiene el mayor flujo de ingreso de información (grado de entrada de 407 pnutos), junto con la del senador Jorge Larrañaga (@jorgewlarranaga). Estas dos cuentas funcionarían como el epicentro de los dos principales conglomerados, si bien la primera abarca una mayor cantidad de vínculos, no siendo una cuenta personal. 
Imagen 3. Red descentralizada de Larrañaga de la campaña \#VivirSinMiedo.

Fuente: elaboración propia en base a modelo de nodos y aristas de tweets y retweets de la campaña \#VivirSinMiedo

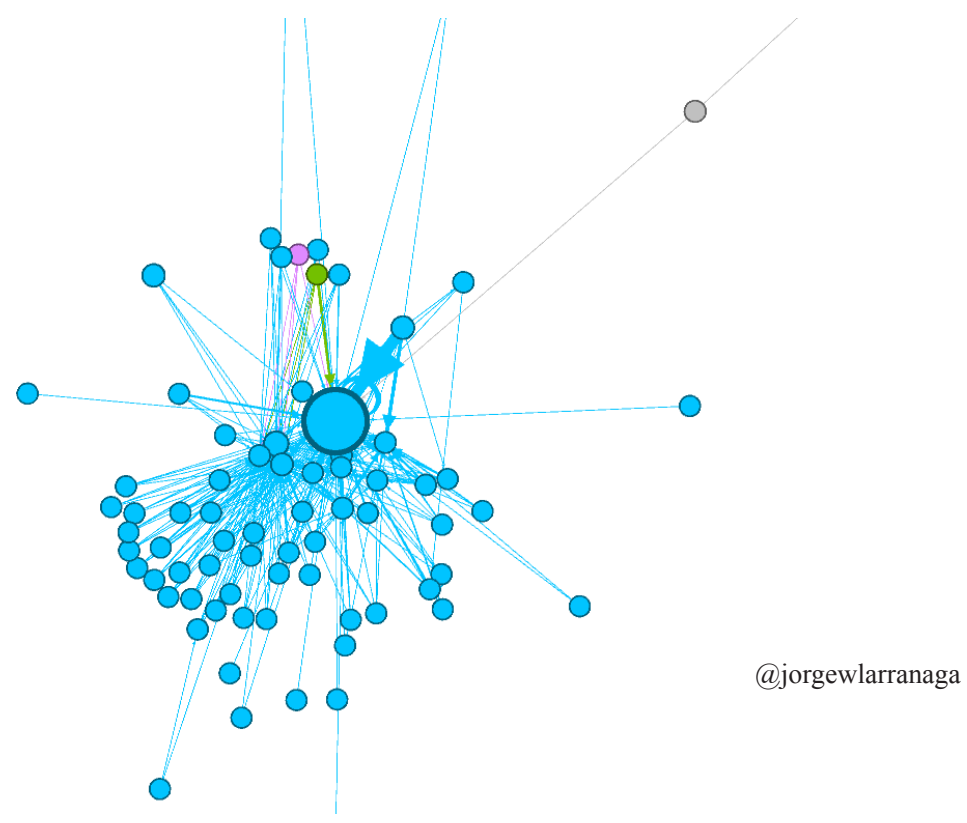

Imagen 4. Red descentralizada de Vivir Sin Miedo de la campaña \#VivirSinMiedo.

Fuente: elaboración propia en base a modelo de nodos y aristas de tweets y retweets de la campaña \#VivirSinMiedo

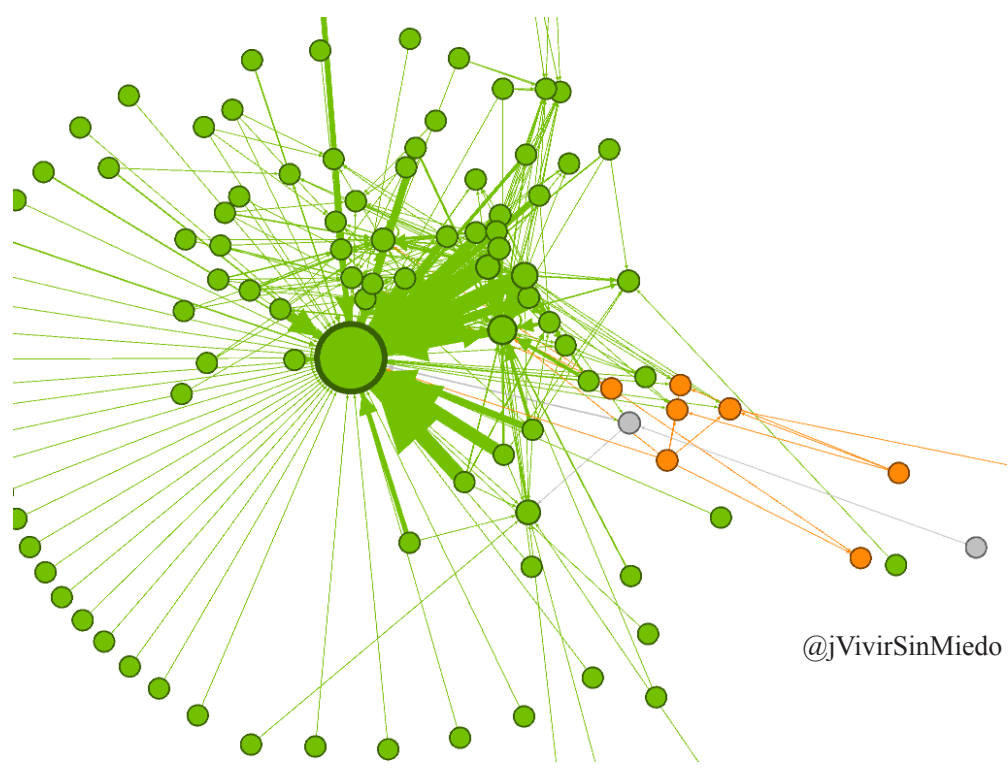


Otro hallazgo relevante atañe a la centralidad y cercanía de estos conglomerados (centralidad de 21818 puntos en el caso de Larrañaga y 19924 en VivirSinMiedo). Es decir, aparecen con mucha frecuencia en muchas de las conexiones cortas del resto de nodos. El protagonismo de estos dos nodos recae en las menciones que realiza el resto de usuarios periféricos a estas dos cuentas y no su viceversa. Por lo que estas últimas no producen información ni son generadoras de opinión en lo que refiere a narrativas políticas (bajo grado de salida de información). Se trata de centros que reciben información, especialmente la cuenta del senador Larrañaga, pero con escasa producción de discurso, ni replica de información sobre el tema. Resulta relevante señalar que la centralidad de Jorge Larrañaga en la campaña \#VivirSinMiedo es producto del status que le confieren los nodos periféricos (la red en general) y no fruto de su esfuerzo personal en posicionarse en el centro de la red como generador de opinión pública.

Respecto a los nodos periféricos de ambas cuentas, los vínculos de Jorge Larrañaga (nodos azules) se componen esencialmente de diputados y senadores que representan a su sector dentro del Partido Nacional (Alianza Nacional). Este cluster resulta el de mayor densidad dentro de la red. Todo el sector de Alianza Nacional se encuentra conectado entre sí, abloquelado a la hora de difundir la campaña \#VivirSinMiedo. Comparten el mismo mensaje entre sí.

Sin embargo, dentro de este "núcleo duro", también se encuentran figuras del Partido Colorado como el senador Pedro Bordaberry, el cual adhirió a la campaña públicamente. Si bien tampoco genera opinión pública ${ }^{18}$, el nodo comparte una parte importante de los vínculos con los correligionarios de Larrañaga, además de encontrarse muy próximo al centro de la red. Ello puede deberse a la proximidad ideológica de ambos senadores, férreos opositores al gobierno del Frente Amplio, y de como el resto de los nodos lo posicionan en cuanto a este tema y su status de oposición.

El nodo VivirSinMiedo se comporta de manera similar a la cuenta de Jorge Larrañaga, básicamente posicionado en el centro, de acuerdo a la entrada de información. Se trata de la campaña institucionalizada más allá de sus adherentes. De allí que no sólo se le confiera un rol central de entrada de información, sino también de generación $^{19}$, teniendo un rol activo en la campaña.

El núcleo duro de la red de Larrañaga lo componen gran parte de la bancada de Alianza Nacional, aunque su periferia en forma de anillo (nodos de color verde) logra expandir la red más allá del núcleo. Este "cinturón" lo conforman adherentes que no tienen conexión directa con Larrañaga, ni conexión entre ellos, pero sí con el Partido Nacional. De allí que el radio de vínculos de VivirSinMiedo sea algo mayor. No obstante, los adherentes más allá de la frontera en la que se encuentran y de sus lazos débiles con los dos nodos centrales, no logran conectar hacia nuevas redes descentralizadas, por lo que pese a su lejanía con el nodo central, no logran expandir la campaña más allá de su núcleo duro. Tienen un rol pasivo dentro de la red. No producen ni replican información. Son meramente interactuados de la cuenta VivirSinMiedo. Y aunque puede presumirse de que estén a favor de la propuesta de campaña, no son claves en su expansión. De caer la cuenta VivirSinMiedo, estos nodos quedarían por fuera de la campaña.

\footnotetext{
Grado de salida de 0 puntos

Grado de salida de 58 puntos
} 
La tercera red descentralizada la componen varios adherentes a la campaña (imagen $n^{\circ} 5$ ), pero que no forman parte de la estructura partidaria. No tienen ningún cargo político y a excepción de su centro, sus nodos no militan activamente. Básicamente son votantes del Partido Nacional y del Partido Colorado, con vínculos débiles hacia la orgánica partidaria. Su nodo centralizado es la cuenta que denominaremos "Usuario_XXX1", militante activo del Partido Nacional. Según sus publicaciones y tónica verbal podría definirse como un usuario anti izquierda y anti Frente Amplio. Al igual que los otros nodos "egos", su grado de salida es menor a su grado de entrada, por lo que no produce activamente narrativa política, más allá de comentar noticias con un tono conservador y por momentos algo agresivo.

Imagen 5. Red descentralizada de Usuario_XXX1 de la campaña \#VivirSinMiedo.

Fuente: elaboración propia en base a modelo de nodos y aristas de tweets y retweets de la campaña \#VivirSinMiedo

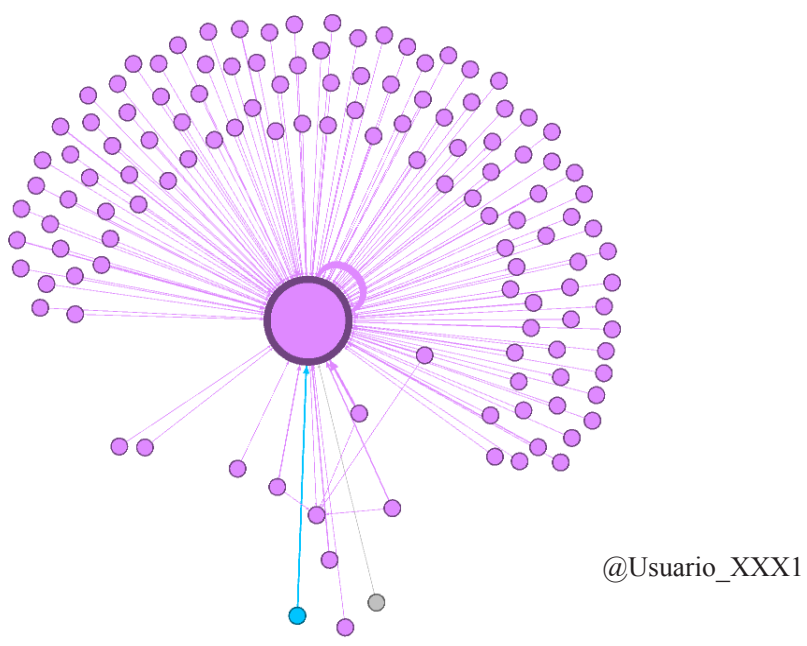

Los nodos periféricos de esta red tampoco se encuentran conectados entre sí y dependen exclusivamente del nodo central, por lo que su interés por la campaña puede resultar muy débil, otorgándole un perfil de bróker (además de ego) a Usuario_XXX1, ya que toda información que entra o sale de estos nodos sobre el hasta \#VivirSinMiedo, necesariamente tiende a pasar por allí. Si bien se muestra críticos con el gobierno, tiene una postura menos agresiva y más reflexiva en cuanto a la inseguridad del país, de allí su lejanía con la red descentralizada de Larrañaga.

Al igual que las redes anteriores, los nodos periféricos de Usuario_XXX1 figuran como frontera de este cluster, sin replicarse el mensaje más allá de los adherentes del Partido Nacional. De la misma forma que el cinturón de nodos de VivirSinMiedo, el rol de estos usuarios también resulta pasivo, limitándose a ser interactuados dentro de la campaña.

Los puentes entre la red de Larrañaga y de VivirSinMiedo junto con la de Usuario_XXX1 lo conforma un cinturón de nodos independientes a la campaña, "brokers", 
nodos, "repartidores" de información (imagen $\mathrm{n}^{\circ} 6$ ), como son periodistas de prensa y radio que replican el hashtag y generan opinión (alto grado de salida), los cuales figuran como intermediarios entre varios clusters. Mencionan el tema, están en la frontera de los núcleos centrales, invitan a debatir, aunque no toman una postura unánime. También se encuentran adherentes que siguen a una parte de la estructura partidaria de Alianza Nacional, pero que también tienen conexiones con la red de Usuario_XXX1.

Imagen 6. Red descentralizada de usuarios independientes de la campaña \#VivirSinMiedo. Fuente: elaboración propia en base a modelo de nodos y aristas de tweets y retweets de la campaña \#VivirSinMiedo

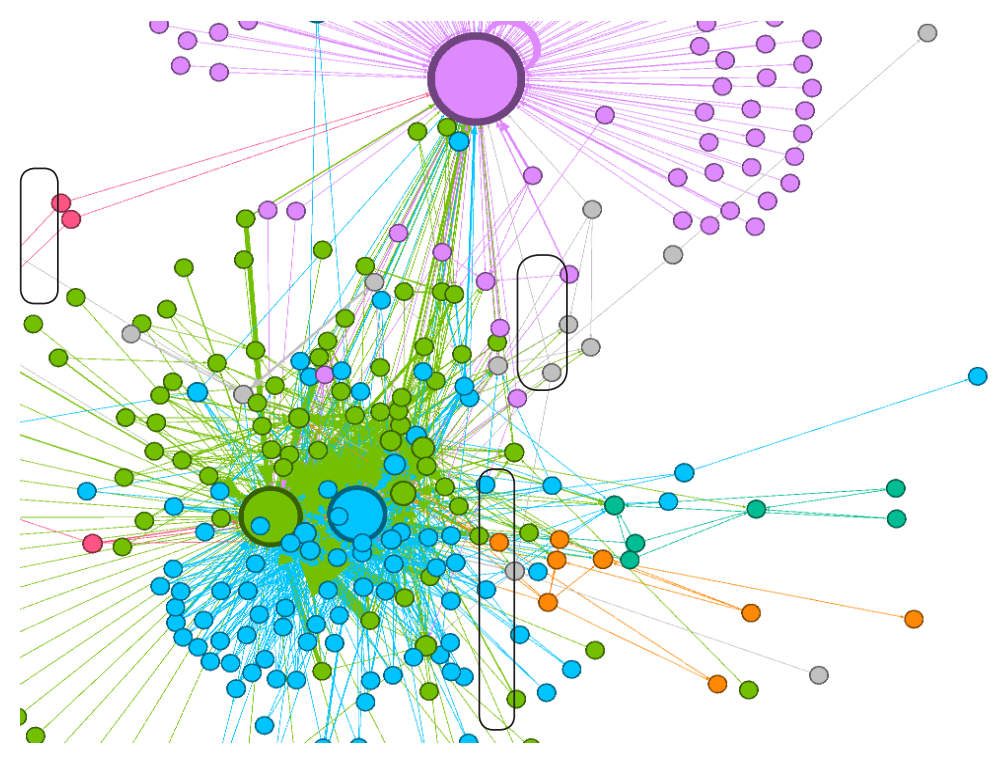

Una cuarta red descentralizada pero de menor expansión es la de AlfTuitea (imagen $\mathrm{n}^{\circ}$ ), es una cuenta no personal, generadora de contenido sarcástico con tono ideológico de izquierda (bromas o reflexiones irónicas). Su única posible conexión con la red central de Larrañaga es a través de la cuenta de Usuario_XXX2. Este último nodo puede definirse como un verdadero bróker entre extremos polares ideológicos (agujero estructural), encontrándose a favor de la campaña \#VivirSinMiedo, pero siendo muy crítica del Partido Nacional, especialmente del sector de Lacalle Pou, principal opositor del gobierno dentro del Partido Nacional, y que no está de acuerdo con la reforma constitucional. Motivo por el cual no figura en la red.

Los opositores a la campaña son muy pocos, por lo que es posible suponer que parte de la estrategia de los usuarios en contra de la reforma sea, no tematizar (mencionar) el hashtag \#VivirSinMiedo, o generar un contra hashtag (\#YoNoFirmo ${ }^{20}$ ) aunque sin mayor éxito, por lo que no figurarían en ninguno de estos espacios. En

20 Aunque se colectaron tweets y retweets de este hashtag, resulto imposible vislumbrar la topología de la red y los puntos de conexión con el hashtag \#VivirSinMiedo ya que \#YoNoFirmo se solapaba con otras campañas por 
este sentido, prácticamente no se encuentra en ninguna de estas redes usuarios con un perfil de izquierda marcado.

Imagen 7. Red descentralizada de AlfTuitea de la campaña \#VivirSinMiedo. Fuente: elaboración propia en base a modelo de nodos y aristas de tweets y retweets de la campaña \#VivirSinMiedo (a)AlfTuitea

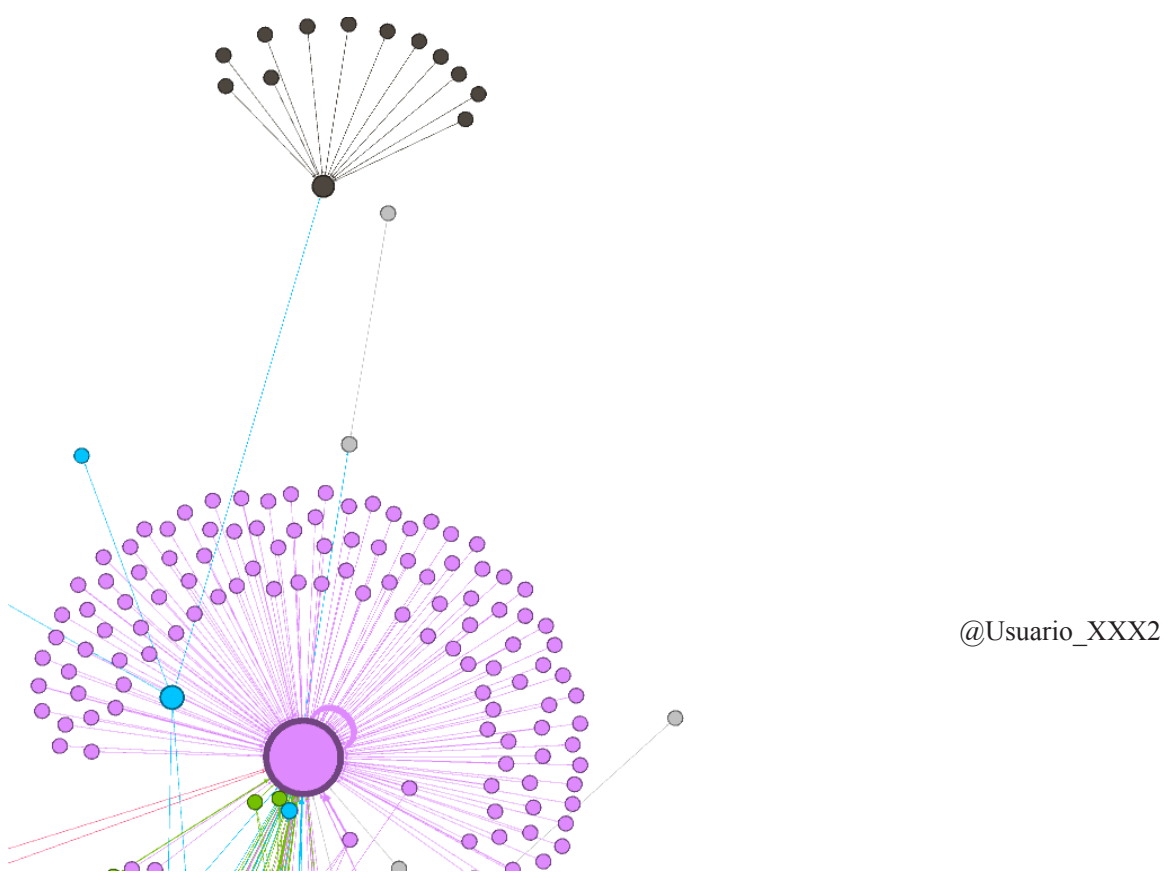

Por último, el resto de nodos por fuera de estas cuatros redes (imagen $n^{\circ} 8$ ), se podrían denominar como pequeñas "islas". Se trata de un espacio (o mejor dicho un abismo) estructural. Alguna vez mencionaron la campaña, pero no comparten ningún vínculo ni con la estructura partidaria, ni con los adherentes a partidos políticos. Aquí figuran medios de comunicación, periodistas y algunos políticos que no tomaron postura, que no adhieren a la reforma, ni replican activamente el tema para construir opinión pública, pero son mencionados para hablar del tema. Como Alvaro Garcé, ex comisionado parlamentario de cárceles, o Daniel Castro, periodista del noticiario Telenoche Cuatro. Figuran en la red de manera involuntaria, o muy periféricamente.

fuera de Uruguay como la de la Agencia de Medio Ambiente y Agua de Andalucía (Amaya, “\#convenioAmaya") en España. 
Imagen 8. Red descentralizada de nodos "islas" de la campaña \#VivirSinMiedo. Fuente: elaboración propia en base a modelo de nodos y aristas de tweets y retweets de la campaña \#VivirSinMiedo


\section{Conclusiones}

La red producto del hashtag \#VivirSinMiedo es el resultado de preferencias partidarias que logran amplificar el mensaje en una parte restringida del espectro político. No resulta producto de conexiones azarosas, sino por el contrario, se reconoce una estructura que refleja gustos y pensamiento compartidos. Difusión y democratización de la información entre "iguales". En este sentido, la hipótesis central de conformación de la red \#VivirSinMiedo como producto de las adherencias partidarias en el mundo off-line tiene cierto asidero, robusteciendo la simpatía que plantea Moreno, como el principio de transitividad de Freeman, estando el senador Jorge Larrañaga en el epicentro de dicha red, aunque de manera involuntaria.

La restricción y limitante de la difusión obedece a su topología principal, netamente descentralizada en tres grandes (sub) grupos. De caer sus nodos centros, cae también la estructura su conjunto. La red la conforman en su núcleo duro diputados y senadores que pertenecen a la estructura partidaria del sector de Larrañaga (Alianza Nacional), replicadores del tema, pero no generadores de opinión pública. Consecuencia de ello no logra expandirse y generar transitividad más allá de estos partidarios. Comparten su postura política entre usuarios iguales o parecidos. Por lo que los flujos de información que generan colocan a Larrañaga de manera emergente en el centro, cohesionando a su grupo partidario de mayor transitividad, pero sin cumplir su cometido último de despolitizar la campaña en procura de lograr un mayor número de adherentes. Por ende, mayor expansión de la red y captación en el espacio virtual.

En cuanto a su difusión, más allá de las fronteras de la estructura partidaria, el conjunto de adherentes votantes también se encuentra sumamente centralizado a 
una parte del espectro ideológico, esencialmente conservador, de derecha y por momentos anti-izquierda o anti - frenteamplista. Si bien ocupan un lugar importante en la red, los lazos que unen a este grupo con la estructura partidaria (cuentas de Larrañaga o seguidores de Vivir Sin Miedo) son sumamente restringidos, marcando la distancia que existe entre la estructura partidaria y sus potenciales votantes.

La distancia en la red con el epicentro de los nodos de Vivir Sin Miedo y Jorge Larrañaga supone también una nueva hipótesis de "distancia ideológica" (algo así como lejanía de las preferencias y gustos, o el mayor nivel de antipatía), reconociéndose a varios adherentes de izquierda muy alejados del centro de la red. Los nodos brokers precisamente serían aquellos que políticamente son de izquierda o neutrales, pero que pueden estar a favor de la reforma constitucional. Sin embargo, los detractores de la campaña, además de ser pocos y estar alejados de los centros de la red, no optan por tematizar la campaña (retwittear el hashtag \#VivirSinMiedo). Por el contrario, se oponen creando un contra hashtag, que en el fondo no hace más que estructurar una nueva red de gustos y preferencias, imposibilitando y polarizando el dialogo entre los diferentes usuarios.

Por último, las fronteras de la red resultan demasiado compactas, dejando un abismo estructural de nodos que actúan en formas de "islas", sin un bróker cercano que conecte la temática sobre todo al centro de la red y al menos polemice sobre la reforma como tal. Muchos de estos nodos resultarían clave para el fin último de Larrañaga, el cual sería además de expandir el mensaje, despolitizar la posible reforma constitucional.

\section{Referencias}

Baran, P. (1964). On distributed Communication. Introduction To Distributed Communication Networks. Santa Mónica California, Estados Unidos. Editor: "The Rand Corporation". Disponible en: https://www.rand.org/content/dam/rand/pubs/research_memoranda/2006/ RM3420.pdf [2019, 25 de enero]

Batlle, A; Cerrillo, A. (2004). Tics y procesos políticos. Barcelona. Documentos Universidad Oberta de Catalunya. XP04/63038/01142

Burt, R, S. (2004). Structural Holes and Good Ideas. Revista The American Journal of Sociology, Volumen 110, $n^{\circ}$ 2, pp. 349-399. Chicago, Estados Unidos. Disponible en: https://www.journals.uchicago.edu/doi/pdfplus/10.1086/421787 [2019, 25 de enero]

Castro Rojas, S. (2012). Ubicuidad y comunicación: los Smartphones. Revista Chasqui. Revista Latinoamericana de Comunicación. $\mathrm{N}^{\circ}$ 118, pp. 91-95. Centro Internacional de Estudios Superiores de Comunicación para América Latina. Quito, Ecuador. Disponible en: https://www.redalyc.org/articulo.oa?id=16057417018 [2019, 25 de enero]

Marín Dueñas, P.P; Díaz Guerra, A. (2016). Uso de Twitter por los partidos y candidatos políticos en las elecciones autonómicas de Madrid 2015. Revista Ámbitos. Revista Andaluza de Comunicación. $\mathrm{N}^{\circ} 32$, pp. 1-16. Universidad de Sevilla. Sevilla, España. Disponible en https://www.redalyc.org/pdf/168/16845702009.pdf [2019, 25 de enero]

Fainholc, B. (2011). Un análisis contemporáneo del Twitter. Revista RED. Revista de Educación a Distancia. $\mathrm{N}^{\circ}$ 26, pp. 1-12. Universidad de Murcia. Murcia, España. Disponible en: https://www.redalyc.org/articulo.oa? $\mathrm{id}=54718738003$ [2019, 25 de enero] 
Freeman, L. C. (1979). Centrality in Social Networks: Conceptual clarification. Revista Social Networks. Volumen 1, N³, pp. 215-39. Editorial Elsevier Sequoia. S.A. Lausana, impreso en Países Bajos DOI: https://doi.org/10.1016/0378-8733(78)90021-7 [2019, 25 de enero]

Ferrás Sexto, C; García, Y. (2009). ¿Son las tecnologías de la información capaces de cambiar las formas de hacer política? Estudio de casos en Galicia. Revista Teoría de la Educación. Educación y Cultura en la Sociedad de la Información. Volumen 10, № 2, pp. 146-164, Universidad de Salamanca. Salamanca, España. Disponible en: https://www.redalyc.org/ articulo.oa?id=201017352010 [2019, 25 de enero]

Gerl, K. (2017). Política 2.0. Internet y el trabajo de los partidos. Revista Nueva Sociedad. $\mathrm{N}^{\circ}$ 269, pp. 61-65. Internacional. ISSN: 0251-355. Disponible en: http://132.248.9.34/hevila/ Nuevasociedad/2017/no269/7.pdf [2019, 25 de enero]

Grupo Radar (2018). El perfil del internauta Uruguayo. $15^{\circ}$ edición. Resumen ejecutivo web. Disponible en: http://www.gruporadar.com.uy/01/?p=2377 [2019, 25 de enero]

Hogan, B. (2017): Online Social Networks: Concepts for Data Colecction and Analysis en Fieldng, N.G; Lee, R; Blank, G. (2017). The Sage Handbook of Online Research Methods. Segunda Edición, pp 241-258. Londres, Inglaterra: Sage. Disponible en: https://papers. ssrn.com/sol3/papers.cfm?abstract_id=3047869 [2019, 25 de enero]

Instituto Nacional de Estadística (2016). Principales resultados Encuesta de Usos de las Tecnologías de la Información y la Comunicación, 2016. Informe. Disponible en: http:// www.ine.gub.uy/c/document_library/get_file?uuid=21edeb0d-0ae1-4bc8-b9a37d515f077228\&groupId=10181 [2019, 25 de enero]

Islas, O. (2008). La sociedad de la ubicuidad. Los prosumidores y un modelo de comunicación para comprender la complejidad de las comunicaciones digitales. Revista Razón y Palabra. Volumen 3, $\mathrm{N}^{\circ}$ 65. Internacional. Disponible en: https://www.redalyc.org/ articulo.oa? id=199520724016 [2019, 25 de enero]

Lusoli, W; Ward, S (2003). Hunting Protestors Mobilisation, Participation, and Protest Online in the Countryside Alliance. Documento presentado en el congreso anual European Consortium for Political Research. Edimburgo, 28 de Marzo - 2 de Abril, 2003. Disponible en: https://pdfs.semanticscholar.org/da40/df57a5c0df557775f603646e26c20c8768cf.pdf [2019, 25 de enero]

Moreno, J, L. (1934). Nervous and mental disease monograph series, no 58. Who shall survive?: A new approach to the problem of human interrelations. Washington, DC, Estados Unidos: Nervous and Mental Disease Publishing Co. Disponible en: https:// archive.org/details/whoshallsurviven00jlmo [2019, 25 de enero]

Orlando D'Adamo; García, V; Kievsky, T. (2015). Comunicación política y redes sociales: análisis de las campañas para las elecciones legislativas de 2013 en la ciudad de Buenos Aires. Revista Mexicana de Opinión Pública. Universidad Nacional Autónoma de México Distrito Federal, México. N¹9, pp. 107-126. Disponible en: https:/www.sciencedirect. com/science/article/pii/S187073001500006X [2019, 25 de enero]

Otaola, L, M (2007). Nuevas Tecnologías de Comunicación: ¿Oportunidad o riesgo para la Esfera Pública y la Democracia?. Revista Razón y Palabra. $\mathrm{N}^{\circ}$ 54. Disponible en: http:// www.razonypalabra.org.mx/anteriores/n54/mlarao.html [2019, 25 de enero]

Radakovich, R; Ponce, M; Morales, M.J; Escuder, S (2013). Trazando un mapa de los medios digitales: Uruguay. Informe Internacional Mapping Media. Open Society Foundations. Disponible en: https://www.opensocietyfoundations.org/sites/default/files/mappingdigital-media-uruguay-sp-20140206_0.pdf [2019, 25 de enero] 
Reveles Vázquez, F (2017). Democracia participativa para el fortalecimiento de la representación política. La experiencia latinoamericana. Revista Andamios Vol 14, №35, pp 71-99. Colegio de Humanidades y Ciencias Sociales, Universidad Autónoma de la Ciudad de México Disponible en: https://www.redalyc.org/articulo.oa?id=62854576004 [2019, 25 de enero]

Salgado Andrade, E. (2013). Twitter en la campaña electoral de 2012. Revista Desacatos, $\mathrm{N}^{\circ} 42$, pp. 217-232. Centro de Investigaciones y Estudios Superiores en Antropología Social. Distrito Federal, México. Disponible en: https://www.redalyc.org/articulo.oa?id=13926971013 [2019, 25 de enero]

Toudert, D. (2014). Evolución de la polarización territorial de la producción Web: ¿Para cuándo la esperada descentralización?. Revista Economía, Sociedad y Territorio. Volumen 14, N 45, pp 549-580. ISSN 2448-6183 Disponible en: http://www.scielo.org.mx/scielo. php?script=sci_arttext\&pid=S1405-84212014000200009 [2019, 25 de enero] 